\title{
Estudio de corrosión galvánica en pares latón/acero inoxidable y latón/fundición de hierro ${ }^{(\cdot)}$
}

\author{
M. Ohanian*, V. Díaz*, M. Corengia*y C. F. Zinola*
}

\begin{abstract}
Resumen
El ataque por corrosión en los sistemas intercambiadores de calor constituye un problema para el mantenimiento de cualquier planta industrial. Se trata de sistemas multigalvánicos con particular complejidad geométrica y fluidodinámica. Las patologías corrosivas incluyen el fenómeno de dealeación selectiva de cinc en las aleaciones de cobre. A fin de explicar un caso particular de ataque por decinficación (deterioro en placa de intercambiador de calor de tubos de inoxidable), el presente trabajo aborda en ensayos a escala de laboratorio, la caracterización e interacciones entre dos aleaciones de cobre y cinc, (Yellow brass -UNS C268- y Admiralty brass -UNS C443-), respecto a acero inoxidable AISI 316 y fundición gris de hierro. Los ensayos se realizan a $20^{\circ} \mathrm{C}$ en disoluciones de $\mathrm{NaCl} 1,5 \%$ y $\mathrm{Na}_{2} \mathrm{SO}_{4} 1,5 \%$ y pH 8 . Se caracterizan electroquímicamente las aleaciones y materiales involucrados mediante barridos potenciodinámicos. Los pares galvánicos formados se analizan mediante el estudio de sus corrientes de acoplamiento. Se concluye acerca de la causa de la patología analizada y de los rangos de potencial de protección de latones y su compatibilidad de acoplamiento con otros metales.
\end{abstract}

\section{A galvanic corrosion study of brass/stainless steel and brass/cast iron couples}

\begin{abstract}
Corrosion attack in heat exchanger systems is a topic of main interest for the maintenance in each industrial plant. These are multigalvanic systems with particular geometric and fluidodynamic complexity. Corrosive damages include zinc selective dealeation in copper alloys. In order to explain zinc dealeation attack, this paper deals with laboratory scale testing, characterization and interactions between two copper and zinc alloys (Yellow brass -UNS C268- and Admiralty brass -UNS C443-) compared to AISI 316 stainless steel and cast iron. The tests were performed at $20{ }^{\circ} \mathrm{C}$ in $1.5 \% \mathrm{NaCl}$ and $1.5 \% \mathrm{Na}_{2} \mathrm{SO}_{4}$ solutions, $\mathrm{pH} 8$ and each material was characterized by potentiodynamic sweeps. The couples are analyzed by studying transient galvanic currents. We conclude about the cause of the analyzed pathology, brass protection potential ranges and its coupling compatibility with other metals.
\end{abstract}

Keywords

Galvanic corrosion; Brass; Copper; Zinc; Dealloying.

\section{INTRODUCCIÓN}

El uso de materiales distintos en la construcción de condensadores de plantas de generación de potencia es una práctica habitual. Lo usual es encontrar condensadores fabricados con su caja de acero al carbono, tubos de titanio, acero inoxidable o aleaciones de cobre y placas de tubos de una aleación de cobre diferente. Las razones para encontrar la diversidad de materiales obedecen a factores mecánicos, de transferencia de calor, de confiabilidad o econó- $\operatorname{micos}^{[1}{ }^{1}$ 2]. En muchas ocasiones la disímil característica de las aleaciones induce una severa corrosión galvánica y particularmente en la placa de tubos ${ }^{[3-7]}$.

La llamada serie galvánica provee una indicación de la tendencia a comportarse como ánodo o cátodo al ponerse en contacto eléctrico un par metálico. La velocidad de corrosión depende de factores tales como la relación de áreas de los electrodos y las resistencias a la polarización de las superficies. Por otra parte la resistencia a la polarización depende de la forma-

(•) Trabajo recibido el día 25 de Agosto de 2010 y aceptado en su forma final el día 11 de Abril de 2011.

* Núcleo Interdisciplinario de Ingeniería Electroquímica, Universidad de la República, José Rodó No 1843, Montevideo, Uruguay. e-mail: mohanian@fing.edu.uy; verodiaz@fing.edu.uy; corengia@fing.edu.uy; fzinola@fcien.edu.uy 
ción o no de películas pasivas, fenómenos de transporte en el electrolito, etc. ${ }^{[8]}$. El comportamiento de acoplamientos galvánicos expuestos en agua de mar de la costa oeste de Suecia, en diferente relación de áreas, es estudiado por Wallén y Andersson mediante la técnica de pérdida de masa ${ }^{[9]}$. Los autores ensayan (entre otros acoplamientos) cuplas de acero inoxidable UNS (American Society of Metals) S31254, con diversas aleaciones de cobre, encontrando que dicho acero causa ataque en todas las aleaciones de cobre y particularmente en la aleación de Muntz. Shams El Din et al. ${ }^{[10]}$ estudian la evolución de potenciales de corrosión de cobre, cinc y aleaciones de estos últimos, en diversas soluciones salinas en condiciones de laboratorio; los autores informan la medida del potencial mixto, al realizar el acoplamiento de metales, teniendo la posición geométrica como parámetro. El-Dahsan et al. ${ }^{[11]}$ estudian en agua de mar del Golfo de Arabia, el acoplamiento de latón al aluminio con titanio y acero inoxidable AISI 316; mediante medidas de potencial de corrosión señalan la evolución de dicho parámetro en el tiempo para los metales individuales y su acoplamiento. Para los acoplamientos, el potencial medido se acerca al del latón. Asimismo los autores reportan corrosión galvánica en menoscabo del latón mediante la medida de la corriente circulante. Marshakov ${ }^{[12]}$ establece velocidades de corrosión por pérdida de masa similares para latones cobre $70 \%$ y cobre $70 \%$ con un $1 \%$ de estaño en condiciones estancas. Dicho autor reporta parámetros de dealeado inferiores para la aleación con estaño. Hodgkiess y Lim $^{[13]}$ desarrollan experiencias de larga duración en acoplamientos de acero inoxidable con aleaciones cuproníquel en agua de mar, investigando la influencia de la temperatura y la presencia de fisuras artificiales; los autores muestran la cantidad de factores que influencian las corrientes de acoplamiento y concluyen acerca de un rol fundamental de la reacción catódica que ocurre en el componente más noble de la cupla.

El proceso de decinficación es un proceso de dealeción selectiva, influenciado por el contenido de cinc en la aleación, el agregado de microaleantes (elementos inhibidores del proceso como estaño, o los más efectivos arsénico, antimonio o fósforo), el $\mathrm{pH}$ de la disolución, y estancamiento de la misma (efecto de bajas velocidad de circulación o formación de fouling). El fenómeno se evidencia por un cambio de color de la aleación, de amarillo a rojo (debido al aumento de la concentración de cobre), pérdida de propiedades mecánicas y pérdida de material en forma horizontal (capas) o formación de pits ${ }^{\text {[14-16] }}$.

Durante la parada por mantenimiento de una de las unidades de producción de energía mediante ciclo térmico de vapor, en UTE (Administración Nacional de Usinas y Transmisiones Eléctricas-República Oriental del Uruguay)-Central Batlle, se pudo apreciar un marcado deterioro de la placa de tubos del lado de agua de enfriamiento. La mencionada planta utiliza el agua de la Bahía de Montevideo, estuario del Río de la Plata. Las características de dicho fluido son extremadamente cambiantes, en cuanto a temperatura (efecto estacional), salinidad y contaminantes (influencia de procedencia del agua: de río u oceánica). El material de la placa de tubos es de latón UNS C280 (Muntz metal) (Cu:Zn, 56/63:balance) ${ }^{\text {[17-19]; }}$ los tubos de intercambio son UNS C443 Admiralty brass (latón de almirantazgo, Cu:Zn:Sn, 71:28:1) o acero inoxidable AISI 316 según la zona de intercambio (los de AISI 316 se disponen en la zona de vacío interna del condensador). En la zona de la placa donde se fijan los tubos de AISI 316 se evidencia la dealeación selectiva de cinc (Fig. 1). En el recambio de tubos se encontraron zonas donde la patología de

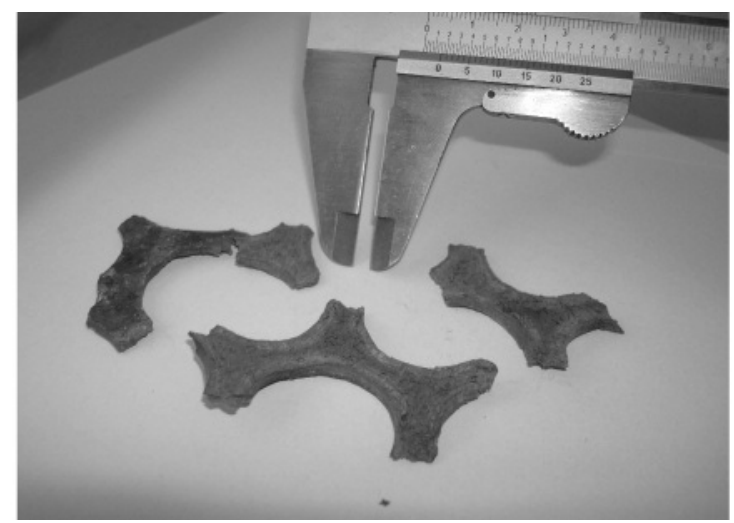

Figura 1. Zona de inserción de tubos de AISI 316 en la placa de latón Muntz (en la fotografía los tubos han sido sacados para recambio). A la derecha, detalle de elementos desprendidos de la placa.

Figure 1. Plate zone where AISI 316 tubes were placed (tubes had been removed for replacement in the photograph). In the right side, elements in detail. 
dealeación del latón alcanza más de cinco milímetros de profundidad. Por otra parte los tubos de AISI 316, han desarrollado patologías corrosivas, con generación de pits, los cuales han generado la condena de la mayoría de los mismos.

El objetivo del presente trabajo es estudiar el fenómeno producido por el acoplamiento de los diferentes materiales a escala de laboratorio, en condiciones similares a las de la entrada al condensador mencionado. La metodología empleada consiste en el empleo de técnicas electroquímicas para caracterizar los materiales individuales y las características de los acoplamientos. Los resultados conducen a la explicación del deterioro encontrado y aportan elementos cuantitativos de decisión para el reemplazo de componentes y adopción de medidas de protección.

\section{MÉTODOS Y MATERIALES}

Las experiencias se realizan a temperatura de $20 \pm 2^{\circ} \mathrm{C}$, en condiciones de agitación proveída por agitador magnético. Los electrolitos empleados son cloruro de sodio $1,5 \%$ (masa/masa) y sulfato de sodio $1,5 \%$ (masa/masa), a pH 8. La aereación de la disolución se realiza en forma natural por la interfase aire/electrolito. Los electrodos de trabajo son pulidos con lija \#220 antes de cada experiencia. Los materiales analizados son AISI 316, fundición gris de hierro, cobre, cinc, Admiralty brass (UNS C443) y Yellow brass (UNS C268). La sustitución de Muntz metal (UNS C280) - material de placa de equipo de condensador-por Yellow brass corresponde a similitud de composición y disponibilidad en el mercado local para generar los electrodos.

Para las experiencias de voltamperometría se usa una celda electroquímica de tres electrodos: electrodo de trabajo, contra-electrodo de platino platinizado y electrodo de referencia de calomel saturado (SCE). La velocidad de barrido es de $5 \mathrm{mV} \mathrm{s}^{-1}$ y los intervalos son entre -1.150 y $500 \mathrm{mV}$. Las ramas -anódica y catódica- se recorren en experiencias independientes, partiendo del potencial de corrosión.

Las experiencias electroquímicas de estudios de pares galvánicos consisten en registros de corriente (entre electrodo de trabajo -latón-y auxiliar -fundición o acero inoxidable-), cada 1 s, en un período experimental de $3.600 \mathrm{~s}$, a potencial fijo de circuito abierto inicial del electrodo de trabajo. Para el caso del AISI 316 se estudia la influencia de su estado superficial sobre el acoplamiento, mediante la aplicación previa de escalones de potencial al acero inoxidable a -200 y $300 \mathrm{mV}$ en cloruro de sodio y 100 y
$300 \mathrm{mV}$ en sulfato de sodio, durante $1.800 \mathrm{~s}$. La elección de los potenciales de tratamiento son realizados a partir de los voltagramas correspondientes, de forma de generar condiciones de pasividad y picado. Las experiencias se realizan con relaciones de área 1/1.

\section{RESULTADOS}

En la figura 2 (arriba) se representan los voltagramas de UNS C268, UNS C443, cobre y cinc en cloruro de sodio 1,5\%. En la figura 2 (abajo) se representa los voltamperogramas de fundición gris y AISI 316 sin tratar en dicho electrolito. En la figura 3 son representados los mismos voltamperogramas en solución de sulfato de sodio $1,5 \%$.

Las cronoamperometrías de los acoplamientos son representadas en las figuras 4 y 5 . La figura 4 corresponde a las corrientes de acoplamiento de latón UNS C443 (Admiralty) en contacto con fundición gris de hierro y aceros inoxidables con diferentes condiciones de superficie: superficie sin tratar y con diferentes tratamientos potenciostáticos a $-0,2 \mathrm{~V}$ y $0,3 \mathrm{~V}$ o $0,1 \mathrm{~V}$ y $0,3 \mathrm{~V}$ según el electrolito de trabajo. En el lado izquierdo de la figura se representan los comportamientos en cloruro de sodio 1,5\% y en el lado derecho es representado el comportamiento en sulfato de sodio. La figura 5 corresponde a idénticas características de la figura 4, pero reportando corrientes de acoplamiento de latón UNS C268 (Yellow brass).

Del análisis de las curvas voltamétricas en cloruro de sodio, se puede expresar que los potenciales de corrosión de electrodos de fundición y AISI 316 ( sin tratar) se encuentran por debajo de los correspondientes a los latones analizados: aproximadamente 0,1 V y 0,4 V para AISI 316 y fundición, respectivamente.

El comportamiento voltamétrico de los latones es intermedio entre sus componentes mayoritarios, aproximándose mucho al del cobre. Su potencial de corrosión se encuentra cercano a los $-0,2 \mathrm{~V}$ vs SCE.

En sulfato de sodio, los potenciales de corrosión de los latones analizados y del AISI 316 son similares, presentando la fundición un potencial de corrosión $0,5 \mathrm{~V}$ menor.

En las experiencias de acoplamiento de materiales, se puede observar que en la cupla UNS C443-AISI 316 el latón actúa como cátodo (corrientes negativas). El tratamiento previo del acero inoxidable influencia la magnitud y trazo de la corriente de acoplamiento, pero siempre en valores negativos.

Difiere el comportamiento del acoplamiento C443/Fundición en ambos electrolitos. Si bien se 


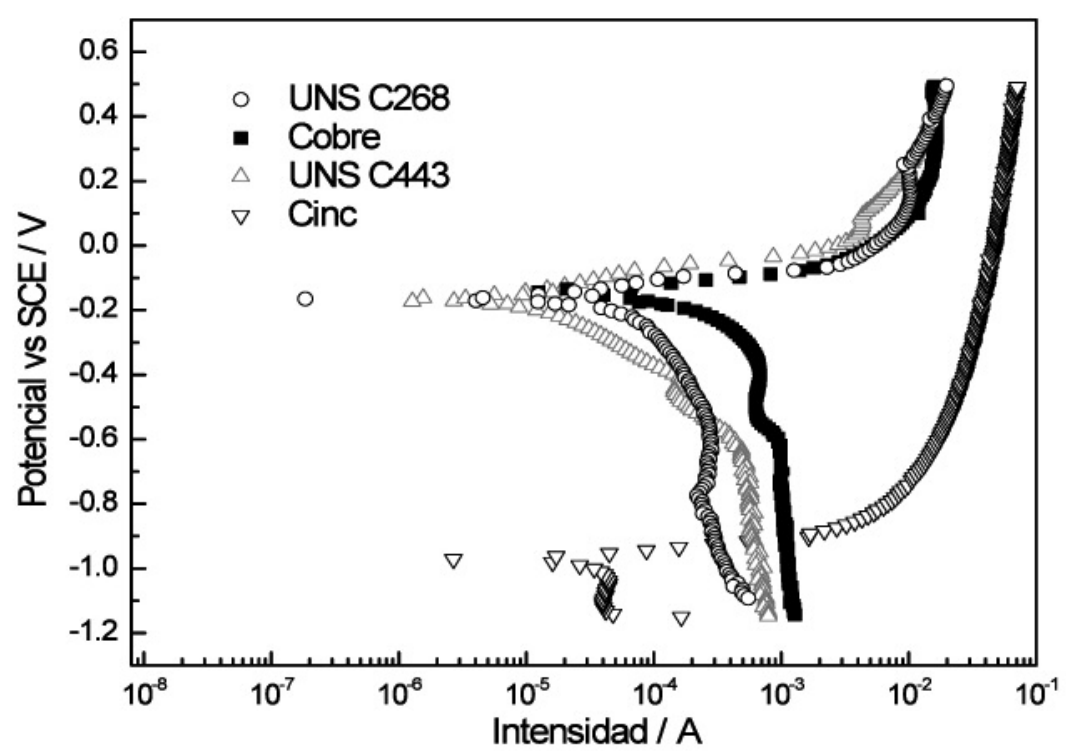

(a)

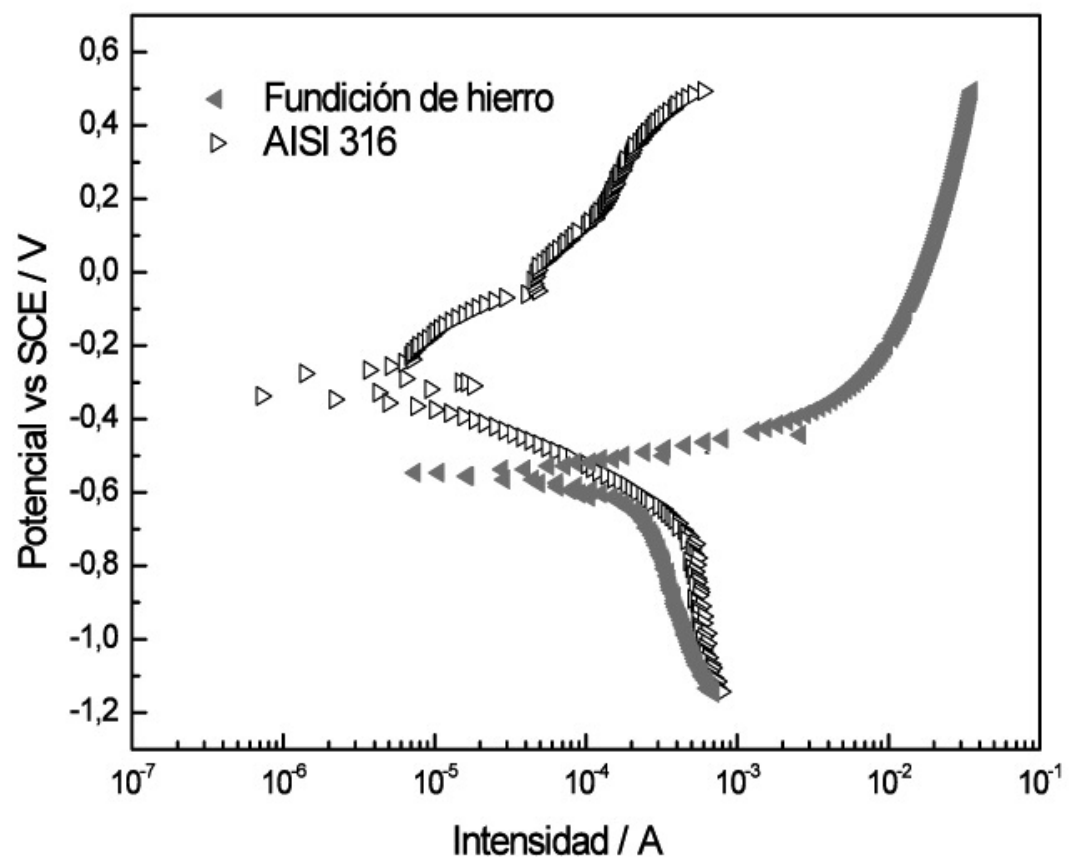

(b)

Figura 2. Arriba se representan los voltagramas de cobre, cinc, UNS C443 y UNS C268 en solución aereada de cloruro de sodio 1,5\%. Abajo se representa el voltagrama de AISI 316 sin tratar y fundición gris de hierro en el mismo electrolito.

Figure 2. Copper, zinc, UNS C443 and UNS C268 voltammograms in aereated $\mathrm{NaCl}$ $1.5 \%$ (up). Cast iron and active AISI 316 voltammograms in the same supporting electrolyte (down).

podrían esperar corrientes superiores a las encontradas para acoplamientos con acero inoxidable (debido a una mayor diferencia de potencial de corrosión entre los electrodos) las corrientes son cercanas a cero y positivas inicialmente en cloruro de sodio (el latón es anódico) y negativas pero inferiores en valor absoluto al par con AISI 316 sin pasivar y pasivado a $300 \mathrm{mV}$ en sulfato de sodio. 


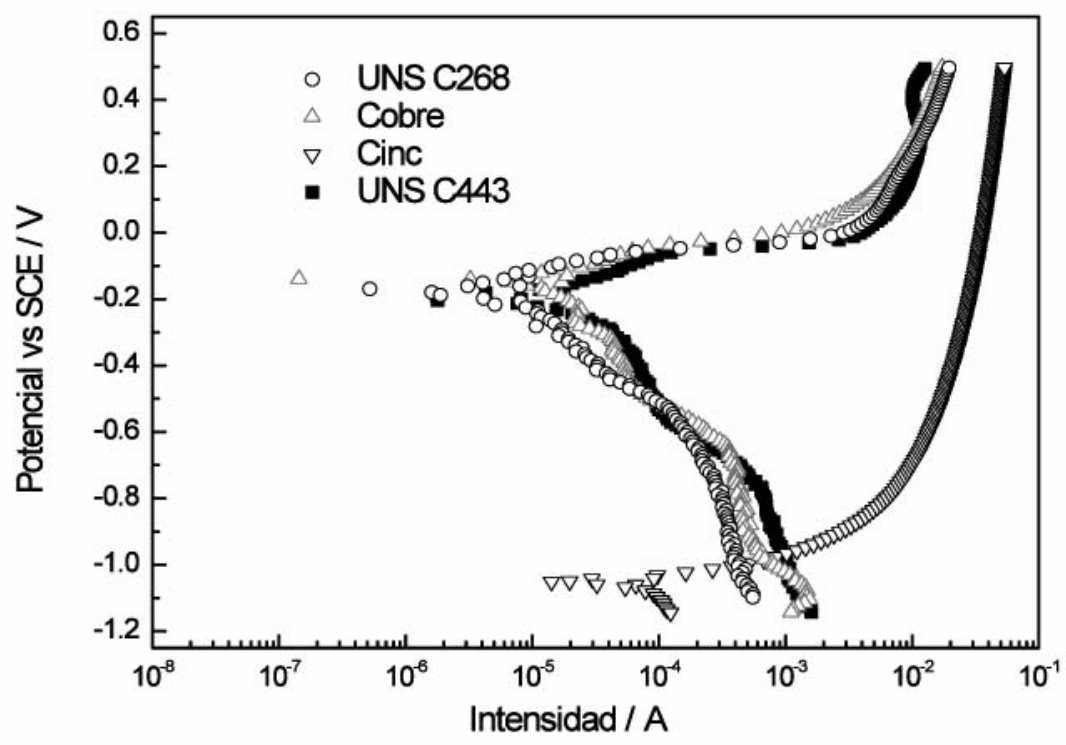

(a)

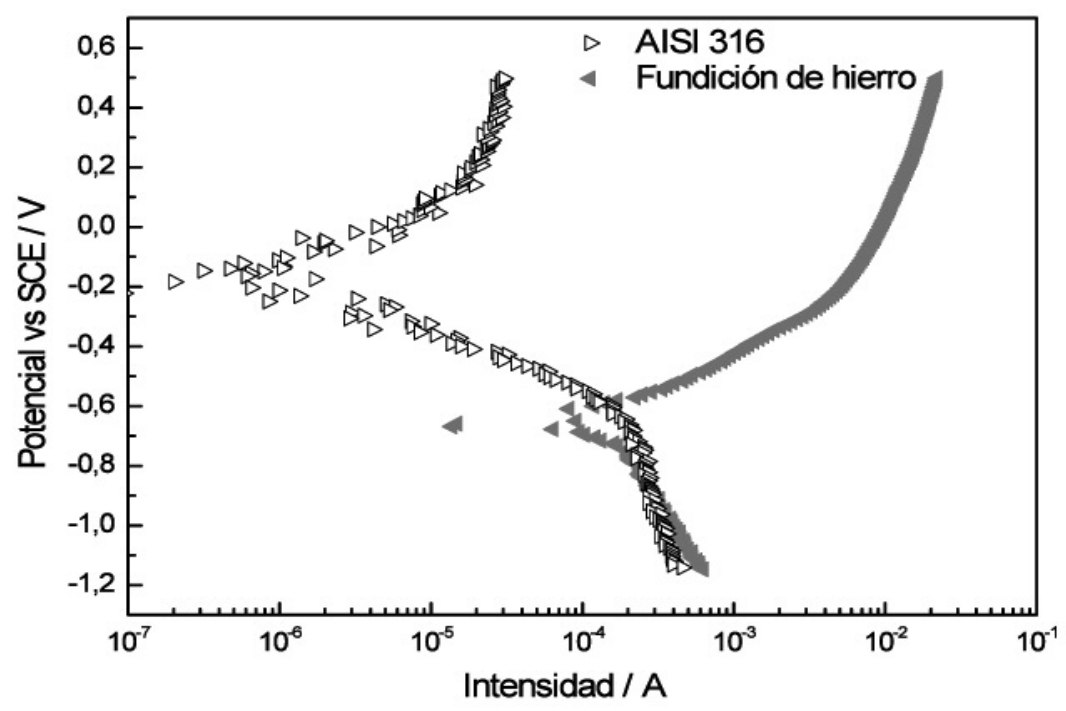

(b)

Figura 3. Arriba se representan los voltagramas de cobre, cinc, latón UNS C443 y UNS C268 en solución aereada de sulfato de sodio 1,5\%. Abajo se representa el voltagrama de AISI 316 sin tratar y fundición gris de hierro en el mismo electrolito.

Figure 3. Copper, zinc, UNS C443 and UNS C268 voltammograms in aereated $\mathrm{Na}_{2} \mathrm{SO}_{4}$ $1.5 \%$ (up). Cast iron and active A/SI 316 voltammograms in the same supporting electrolyte (down).

Para el caso del latón UNS C268 (Yellow brass) el comportamiento frente al acoplamiento es diferente al encontrado con el electrodo de C443; las corrientes de acoplamiento ubican siempre como anódico al latón, siendo la corriente del acoplamiento con fundición sensiblemente superior al resto de las encontradas.

\section{DISCUSIÓN}

La diferencia de comportamiento entre ambos latones se puede atribuir a dos aspectos: por un lado la inclusión de estaño y arsénico como aleantes minoritarios en el UNS C443 y la diferente relación de los componentes mayoritarios de la aleación. 


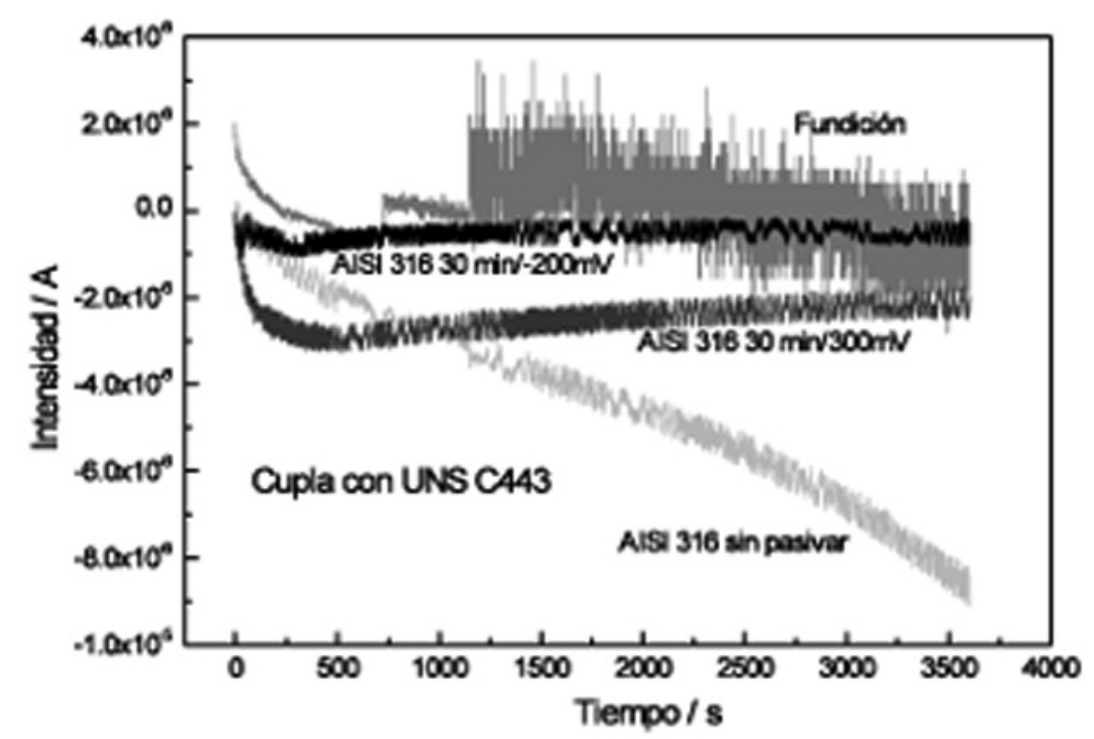

(a)

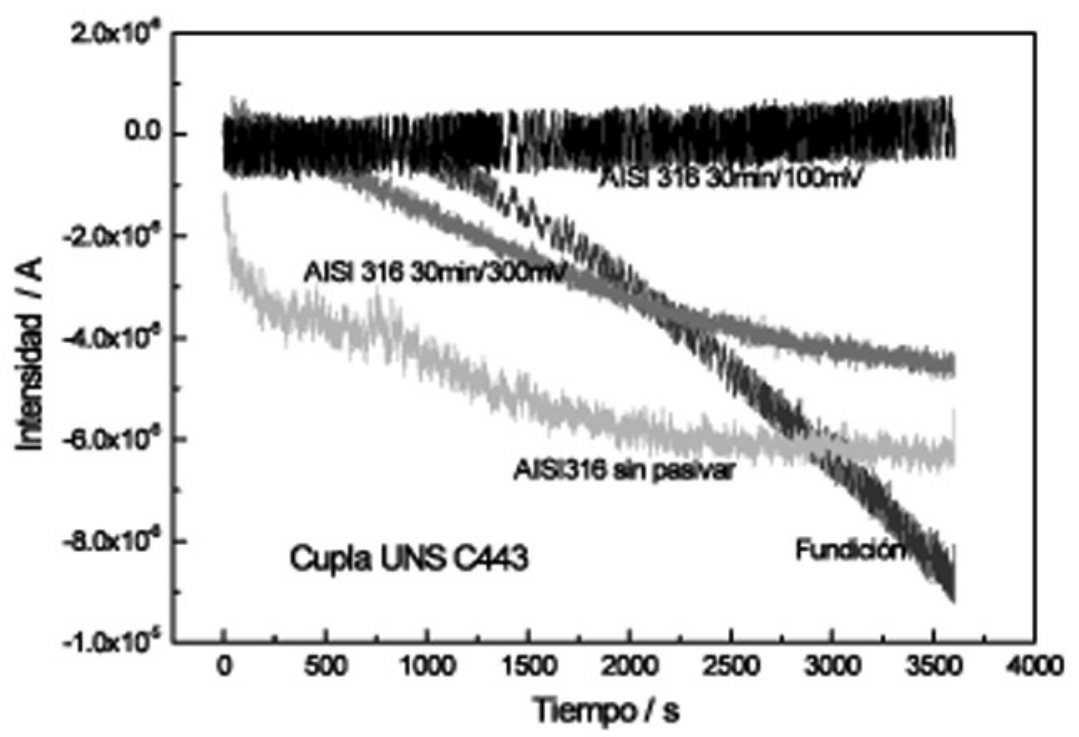

(b)

Figura 4. Corrientes de acoplamiento entre UNS C443 en contacto con fundición de hierro y AISI 316 en diferentes condiciones de superficie, para electrolitos cloruro de sodio $1,5 \%$ (arriba) y sulfato de sodio $1,5 \%$ (abajo).

Figure 4. UNS C443/ cast iron and UNS C443/AISI 316 (active and passive) transient galvanic currents in $\mathrm{NaCl}$ and $\mathrm{Na}_{2} \mathrm{SO}_{4} 1.5 \%$ as supporting electrolyte.

El comportamiento de los latones en medio acuoso puede analizarse a través de sus correspondientes voltamperometrías ${ }^{[20-23]}$. Realizando un barrido desde potenciales catódicos hacia el potencial de corrosión, la disolución inicial forma $\mathrm{Zn}^{+2}$ a aproximadamente $-1.1 \mathrm{~V}$ vs. SCE. A potenciales mayores (aproximadamente $-0,6 \mathrm{~V}$ vs. SCE) se oxida el cobre -conjuntamente con el cinc- y si el contraión es clo- ruro, se forma el $\mathrm{CuCl}$ insoluble en la superficie y el $\mathrm{CuCl}_{2}{ }^{-}$en disolución.

$$
\begin{aligned}
& \mathrm{Cu}+\mathrm{Cl}^{-} \rightarrow \mathrm{CuCl}_{\text {ads }}+\mathrm{e}^{-} \\
& \mathrm{Cu}+2 \mathrm{Cl}^{-} \rightarrow \mathrm{CuCl}_{2}^{-}+\mathrm{e}^{-}
\end{aligned}
$$




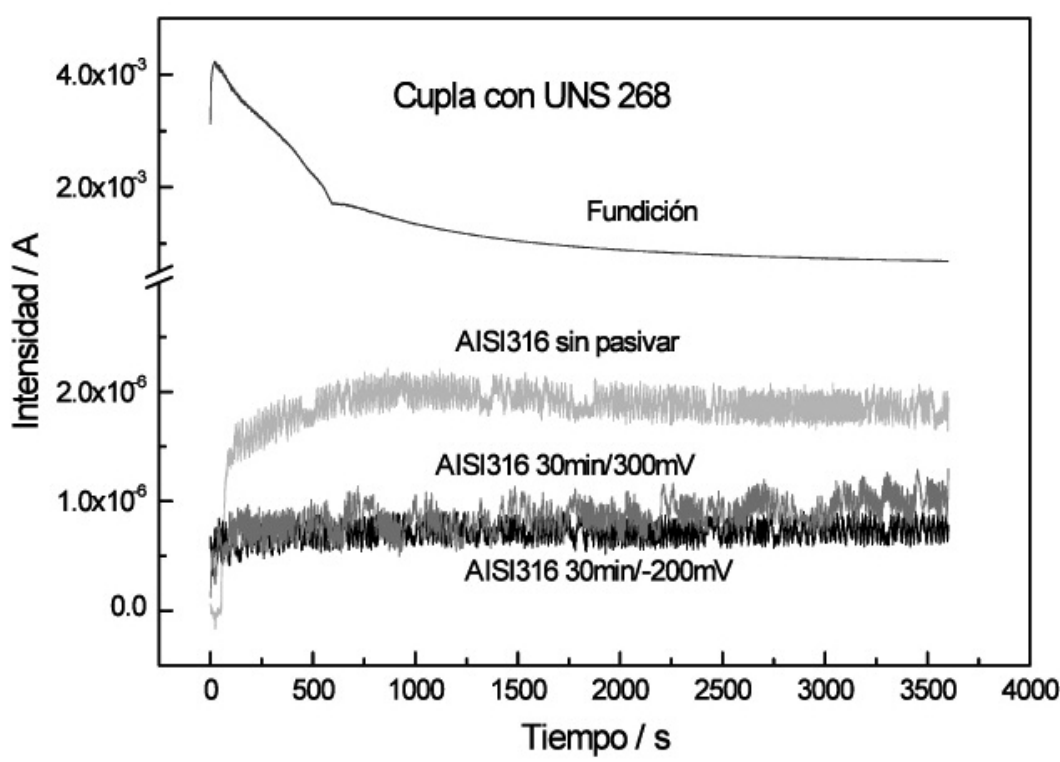

(a)

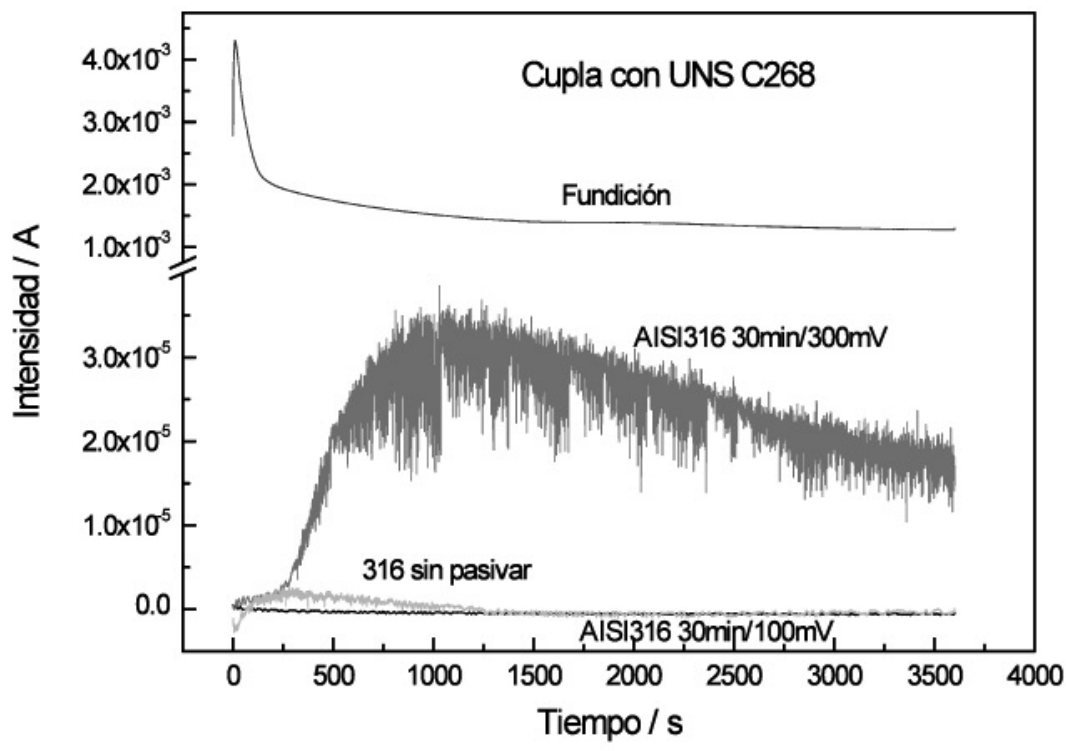

(b)

Figura 5. Corrientes de acoplamiento entre UNS C268 en contacto con fundición de hierro y AISI 316 en diferentes condiciones de superficie, para electrolitos cloruro de sodio $1,5 \%$ (arriba) y sulfato de sodio $1,5 \%$ (abajo).

Figure 5. UNS C268/ cast iron and UNS C268 /AISI 316 (active and passive) transient galvanic currents in $\mathrm{NaCl}$ and $\mathrm{Na}_{2} \mathrm{SO}_{4} 1.5 \%$ as supporting electrolyte.

La disolución de cobre aumenta su concentración, y se produce el intercambio $\mathrm{Cu} / \mathrm{Zn}$, como protección catódica dada por el cinc, incrementándose la concentración de cobre en la superficie metálica.

$$
2 \mathrm{CuCl}_{2}^{-}+\mathrm{Zn} \rightarrow 2 \mathrm{Cu}+\mathrm{ZnCl}_{4}^{2-}
$$

A potenciales mayores a $0 \mathrm{~V}$, el aumento de la disolución de cobre no es compensado por la pro- tección del cinc, mientras que entre $0,2 \mathrm{~V}$ y $0,4 \mathrm{~V}$ ocurre la oxidación a $\mathrm{Cu}^{2+}$.

En sulfato de sodio el comportamiento difiere al explicitado para el medio con cloruros, ya que no existe la posibilidad de formación de compuestos insolubles de cobre. Bajo estas condiciones de $\mathrm{pH}$ y a potenciales inferiores a $-0,25 \mathrm{~V}$, se puede considerar que el cobre permanece inmune mientras el cinc se disuelve: 


$$
\mathrm{Zn} \rightarrow \mathrm{Zn}^{2+}+2 \mathrm{e}^{-}
$$

Además, se puede establecer al $\mathrm{pH}$ de trabajo el pasaje de cinc a hidróxido, el cual puede generar cierto grado de protección en la superficie:

$$
\mathrm{Zn}^{2+}+2 \mathrm{H}_{2} \mathrm{O} \rightarrow \mathrm{Zn}(\mathrm{OH})_{2}+2 \mathrm{H}^{+}
$$

Asimismo, los átomos de cinc pueden difundir hacia el electrolito, dejando vacancias en la red metálica:

$$
\mathrm{CuZn} \rightarrow \mathrm{Cu}^{*}<>+\mathrm{Zn}^{2+}+2 \mathrm{e}^{-}
$$

Donde $\mathrm{Cu}^{*}<>$ representa el estado coordinado del cobre en la red y la vacancia respectiva en ésta ${ }^{[24]}$.

Las consideraciones anteriores reflejan la química de un latón con sólo cobre y cinc como constituyentes. Respecto al efecto de microaleantes como inhibidores de decinficación, Kondrashin establece que el estaño podría llegar a tener efecto sobre el dealeado del latón, pero el mismo no sería tan marcado como el producido por el arsénico. Dicho autor menciona dos mecanismos que involucran al arsénico y que pueden extenderse para la acción del estaño como elemento para disminuir la decinficación. La primer teoría en la disminución del dealeado se basa en el concepto del efecto bloqueante del agente aleante minoritario en la superficie metálica. En el caso del latón UNS C443, podemos asignar el papel de inhibir la redeposición de cobre a la formación de capas de $\mathrm{As}_{2} \mathrm{O}_{3}$ o de $\mathrm{SnO}_{2}$ y como resultado se inhibe la disolución anódica. La otra teoría propuesta involucra un ciclo de reacciones redox en fase heterogénea, con pasaje de iones cúpricos a cuprosos en solución, los cuales tendrían menor tendencia a termodinámica a la reducción ${ }^{[24]}$.

El otro aspecto mencionado como diferencia entre los dos latones analizados es la diferencia de composición de los aleantes mayoritarios: cabe recordar que el latón UNS C268 es más rico en cinc, el metal más electronegativo de los analizados y esto incide en la tendencia de corrientes reportadas.

El acoplamiento de dos metales inmersos en un electrolito genera un par galvánico. Este comportamiento se explica mediante la teoría del potencial mixto, con -al menos- cuatro reacciones participantes, las dos anódicas y las catódicas presentes en los dos electrodos. El potencial y la corriente de corrosión resultante depende, entre otros factores, de la relación de áreas, de fenómenos de transporte de masa, de la distribución de corriente, la formación de películas pasivas, etc. En el caso en estudio se debe considerar que existen tres posibles reacciones anó- dicas, dos de ellas (electrooxidación de cobre y cinc) pueden ocurrir sobre el latón y la restante en el contraelectrodo (electrooxidación del hierro). Para el latón UNS C443 (Admiralty) hay una cuarta reacción anódica posible (la electrooxidación del aleante minoritario) capaz de generar una capa de productos de corrosión posiblemente más compacta. Para el acero inoxidable existe otra reacción anódica posible y es la correspondiente al cromo, la que igualmente puede generar películas pasivas. La reacción catódica es la reducción de oxígeno (suponiendo despreciable la evolución de hidrógeno en el $\mathrm{pH}$ de trabajo) y es pasible de darse en ambos electrodos, pero su velocidad depende de la superficie en la cual se genere. La influencia de todas estas condiciones determina la reacción predominante que se produce en el latón, el cual es susceptible de desarrollar dealeación selectiva en tanto se encuentre por encima del potencial de corrosión del cinc y por debajo del de cobre.

Para el caso del latón UNS 268, las corrientes anódicas del acoplamiento se pueden atribuir al pasaje de cinc a solución, componiéndose la reacción catódica (en forma mayoritaria) en el contraelectrodo. En el acoplamiento con fundición de hierro el efecto es muy notorio y contrapuesto a lo que primeramente se podría esperar al -tan solo- comparar los potenciales de corrosión de los electrodos. En el acoplamiento con acero inoxidable, si bien éste continúa comportándose como dominantemente catódico, no se generan las corrientes tan altas vistas con el acoplamiento a fundición, posiblemente por una menor velocidad de la reacción catódica en la superficie de inoxidable. Por otra parte, existe una influencia del estado superficial inicial del AISI 316.

El comportamiento del UNS C443 refleja la formación de una película pasiva en la superficie del latón, dicha película actúa como resistencia anódica del zinc, por lo que se dan las reacciones catódicas preponderantemente en la superficie del latón.

La situación de acoplamiento latón UNS C268/acero inoxidable es la que se informa en las tradicionales series galvánicas ${ }^{[25]}$. La cupla UNS C443/acero inoxidable se contrapone a lo informado en dichas series. Si el acero inoxidable se encuentra en estado activo, el mismo es aún más anódico respecto al latón UNS C443. En el caso de que el tratamiento se realice a potenciales en los cuales se genera picado, el comportamiento se acerca al del estado sin tratar. El acero inoxidable en condiciones superficiales 'no pasivas' está presente en tanto existan mecanismos despasivantes: presencia de cloruros, microorganismos, etc. [26 y 27]. 


\section{CONCLUSIONES}

- Las técnicas de medida de corriente de pares conjuntamente a barridos potenciodinámicos permiten tener una visión de los complejos sistemas de trabajo. La medida única de voltamperometría en el caso de latones en su zona catódica, puede encubrir una disolución del elemento más electronegativo.

- Desde un punto de vista tecnológico, estos resultados permiten definir el potencial al cual proteger a una pieza de latón. En caso de aplicarse protección catódica, es necesario hacerlo por debajo del potencial del cinc se asegura no oxidar dicho elemento, aunque ello es impracticable en toda la superficie de elementos con gran tamaño o con complejidad geométrica. Por otra parte puede significar un gasto de corriente excesivo o complicaciones por sobreprotección: ataque cáustico o fragilización por hidrógeno. Un nivel de trabajo razonable puede ser a potenciales más negativos a $-0,6 \mathrm{~V}$ vs. SCE., en este potencial aún no se forman iones cuprosos y por tanto no se genera el mecanismo de reposición e intercambio con el cinc. En todo caso se debe facilitar la formación de un film pasivo en la superficie del latón, tanto sea a través de un manejo de $\mathrm{pH}$ (protección por formación de capa de hidróxido de cinc), teniendo un microaleante en el latón (estaño y arsénico en C443) o la dosificación de sales ferrosas ${ }^{[28 \text { y } 29]} \mathrm{u}$ otros inhibidores ${ }^{[30-32]}$.

- Por otra parte, las experiencias de acoplamiento realizadas aportan información valiosa referente a la elección de materiales de sustitución durante la parada por mantenimiento. Se recomienda en el caso práctico mencionado, la sustitución de los tubos de AISI 316 por Admiralty brass (UNS C443) en la zona de vacío del condensador, para prevenir el par en la placa de latón Muntz (UNS C280). Se advierte, respecto a lo anterior, acerca del cuidado en la dosificación excesiva de hidracina (inhibidor de corrosión de hierro en caldera), la cual puede generar amoníaco, y atacar los tubos del lado del condensado ${ }^{[33]}$.

\section{Agradecimientos}

Departamento de Mantenimiento, CCTT, UTE: Ing. Leonardo Paulerci y Técnico Agustín Perotti.

\section{REFERENCIAS}

[1] A. Duran, J.L. Pérez-Rodríguez, L.K. Herrera, M.C. Jiménez-de-Haro, M.D. Robador, A. Justo, J.M. Blanes y J.C. Pérez-Ferrer, Rev. Metal. Madrid 44 (2008) 85-91.

[2] D. M. Bastidas, I. Cayuela and J. M. Bastidas, Rev. Metal. Madrid 42 (2006) 367-381.

[3] R. K. Pandey, Eng. Fail. Anal., 13 (2006) 739-746

[4] J. A. Beavers, A. K. Agrawal and W. E Berry, J. Materials for Energy Systems, 4 (3) (1982) 168-182

[5] J.R. Scully, H.P. Hack (Eds.), Galvanic Corrosion, ASTM, 2002, pp. 136-157.

[6] R. Francis, Br. Corros. J. 22 (1987) 199-201.

[7] W. Kirk, http://www.copper.org/applications/ cuni/txt_condenser_heat_exch_syst.html

[8] J.R. Scully, H.P. Hack (Eds.), Galvanic Corrosion, ASTM, 2002, pp. 1-12.

[9] B. Wallén and T. Andersson, ACOM, No 2, 1987, pp. 1-7.

[10] A. M. Shams El Din, J. M. Abd El Kader and M. M. Badran, Br. Corros. J. 16 (1981) 32-37.

[11] M.E. El-Dahshan, A.M. Shams El Din and H.H. Haggag, Desalination 142 (2002) 161-169.

[12] I. K. Marshakov; Protection of Metals 41 (2005) 227-233.

[13] Hodgkiess and C. W. Lim, Corros. Sci. 34 (1993) 269-283.

[14] H. M. Herro and R. D. Port, The Nalco Guide to Cooling Water System Failure Analysis, Nalco Chemical Company, Mc Graw Hill, EE.UU., 1993, pp. 295-311.

[15] L. L. Shreir, R. A. Jarman, G. T. Burstein, Corrosion, Butterworth Heinemann, Tercera Ed., Oxford, Gran Bretaña, 1995, pp. 4:38-4:75

[16] Z. Xia and Z. Szklarska-Smialowska; Corrosion (46) 85-88.

[17] A. M. Shams El Din; Desalination 93 (1993) 487-498.

[18] A. M. Shams El Din; Desalination 93 (1993) 499-516.

[19] P. R. Roberge, Handbook of Corrosion Engineering; Mc Graw Hill, 1999, pp. 1.072-1.074.

[20] M. Kabasakaloglu, T. Kiyac, O. Sendil and A. Asan, Appl. Surf. Sci. 193 (2002) 167-174

[21] F. M. Al-Kharaffi, B. G. Ateya and R. M. Abd Allah, J. Appl. Electrochem. 34 (2004) 47-53.

[22] K. Balakrishan and V.K. Venkatesan, Electrochimica Acta 24 (1979) 131-138.

[23] B. S. Kim, T. Piao, S. N. Hoier and S. M. Park, Corros. Sci. 37 (1995) 557-570.

[24] V. Yu. Kondrashin, Protection of Metals 41 (2005) 138-145. 
[25] J. A. González, Teoría y Práctica de la Lucha Contra la Corrosión, CENIM, España, 1984, pp. 30-33.

[26] A. U. Malik, N. A. Siddiki and I. N. Andijani, Desalination 97 (1994) 189-197.

[27] H. A. Videla, International Biodeterioration \& Biodegradation (1994) 245-257.

[28] R. F. North and M. J. Pryor, Corros. Sci. 8 (1968) 149-157.

[29] J. M. Popplewell, R. J. Hart and J. A. Ford, Corros. Sci. 13 (1973) 295-309.
[30] F. J. Presuel-Moreno, M. A. Jakab and J. R. Scully, J. Electrochem. Soc. 152 (2005) B376B387.

[31] R. B. Faltermeier, Studies in Conservation, 44 (1998) 121-128.

[32] T. Kosec, I. Milosev and B. Pihlar, Appl. Surf. Sci., 253 (2007) 8.863-8.873.

[33] L.L. Shreir, R.A. Jarman and G.T. Burstein, Corrosion, Butterworth Heinemann, Tercera Ed., Oxford, Inglaterra, 1995, pp. 17:67 - 17:93. 